\title{
AMPLIFICATEURS LOGARITHMIQUES A TRANSISTORS M.O.S. POUR INSTRUMENTATION SPATIALE
}

\author{
Par Alain LIÉGEOIS, \\ Laboratoire de Génie Électrique, Toulouse.
}

\begin{abstract}
Résumé. - L'étude qui fait l'objet de cet article concerne les caractéristiques des transistors Métal-Oxyde-Semiconducteur et des transistors plans au silicium fonctionnant à très faible niveau, qui permettent la conception d'amplificateurs logarithmiques à composants solides pour l'instrumentation spatiale. La plupart des résultats sont illustrés par la mise en œuvre d'un dispositif expérimental destiné à la mesure de courants compris entre $10^{-12}$ et $10^{-8} \mathrm{~A}$.

L'auteur qui expose tout particulièrement les problèmes relatifs à l'obtention d'une faible constante de temps, d'une réponse logarithmique et de faibles dérives décrit les solutions apportées et en définit les limites d'utilisation.
\end{abstract}

Abstract. - The subject of this paper concerns chiefly the characteristics of metal-oxydesemiconductor transistors and low level silicon planar transistors, which can be used to design solid-state logarithmic amplifiers for space experiments. The main properties of these components have been used to develop an experimental device for measurements of currents over the range $10^{-12} \mathrm{~A}$ up to $10^{-8} \mathrm{~A}$.

A fast transient response, a logarithmic transfer law and low drifts are achieved by the author who describes the solutions given to the problems and determines the limitations.

Introduction. - L'importance prise depuis quelques années par les techniques spatiales et nucléaires exige une instrumentation de mesure et de contrôle rapide, très précise et très fiable. Ces techniques font souvent appel à l'électrométrie pour l'amplification des très faibles courants issus de détecteurs haute

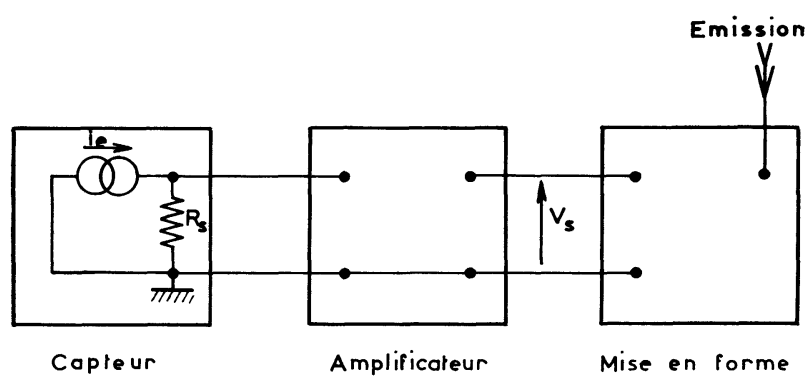

FIG. 1. - Chaîne de mesure.

impédance, spectromètres de masse ou chambres d'ionisation. Dans le cas de l'instrumentation spatiale, il est souvent nécessaire que la tension de sortie $V_{\mathrm{s}}$ des amplificateurs soit une fonction concave du courant $i_{\mathrm{e}}$ à mesurer (réponse " comprimée » assurant une commutation automatique de gain); une plage étendue de niveaux du signal d'entrée est ainsi transformée en une gamme compatible avec les possibilités du système d'enregistrement ou de télémesure ( fig. 1). Les circuits doivent de plus être compacts et légers, et présenter une grande stabilité de caractéristiques malgré les contraintes d'environnement (température, radiations, etc.) imposées par chaque mission.
Le développement technologique des composants à semiconducteurs a conduit récemment à l'élaboration d'éléments actifs à haut isolement d'entrée, les transistors Métal-Oxyde-Semiconducteur (M.O.S.) possédant a priori des qualités requises par les expériences d'électrométrie spatiale. Des études particulières de la fiabilité de composants et de circuits, effectuées au Laboratoire de Génie Électrique de Toulouse, ont cependant été nécessaires pour conduire à la réalisation d'amplificateurs logarithmiques à transistors M.O.S. présentant les moindres dérives sous l'action des variations des paramètres qui définissent l'environnement spatial.

1. Schéma de principe de l'amplificateur. - La structure adoptée ( $f g .2$ ) possède les avantages d'un

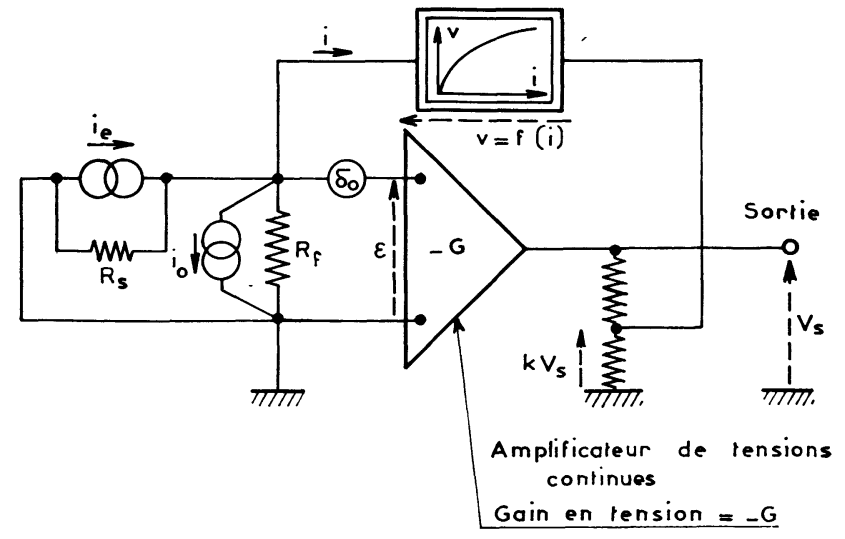

FIG. 2. - Schéma de principe. 
faible temps de réponse, d'une impédance d'entrée et d'une impédance de sortie réduites :

$R_{\mathrm{s}}$ est la résistance d'isolement du capteur,

$R_{\mathrm{f}}$ est la résistance d'isolement de la chaîne directe de l'amplificateur,

$G$ est son gain en tension,

$i_{0}$ représente le courant d'entrée de la chaîne directe,

$\delta_{0}$ est la source de tension équivalente aux dérives de cette chaîne directe,

Le circuit de contre-réaction (figuré ici par un dipôle) a pour caractéristique $v=f(i)$.

Le fonctionnement en régime continu du circuit de la figure 2 est alors décrit par l'équation :

$K V_{\mathrm{s}} \cdot\left[1+\frac{1}{K G}\right]-\delta_{0}$
$+f\left(i_{\mathrm{e}}-i_{0}+\frac{V_{\mathrm{s}}}{G} \cdot\left[\frac{1}{R_{\mathrm{f}}}+\frac{1}{R_{\mathrm{s}}}\right]-\delta_{0} \cdot\left[\frac{1}{R_{\mathrm{f}}}+\frac{1}{R_{\mathrm{s}}}\right]\right)=0$.

Dans le cas idéal où l'amplificateur de la chaîne directe est supposé sans dérives, sans courant d'entrée et de gain infini, la relation (1) conduit à :

$$
V_{\mathrm{s}}=-\frac{1}{K} f\left(i_{\mathrm{e}}\right) \text {. }
$$

En particulier, si la caractéristique de l'élément de contre-réaction est $v=a+b \log i$, où $a$ et $b$ sont des constantes, la tension $V_{\mathrm{s}}$ de sortie de l'amplificateur est une fonction linéaire du logarithme du courant $i_{\mathrm{e}}$ à mesurer.

En pratique cependant, la relation (1) souligne le rôle fondamental joué par $i_{0}, \delta_{0}$ et $R_{\mathrm{f}}$, même lorsque le gain $G$ est de très grande valeur. En effet, (1) devient dans ce cas :

$$
K V_{\mathrm{s}}-\delta_{0}=-f\left(i_{\mathrm{e}}-i_{0}-\delta_{0}\left[\frac{1}{R_{\mathrm{f}}}+\frac{1}{R_{\mathrm{s}}}\right]\right) .
$$

L'équation (3) fait apparaître une erreur « de lecture $» \delta_{0}$ et une erreur « de mesure » $i_{0}+\delta_{0}\left[\frac{1}{R_{\mathrm{f}}}+\frac{1}{R_{\mathrm{s}}}\right]$. Or, $i_{0}$ est le courant d'entrée du premier étage d'amplification de la chaîne directe, $R_{\mathrm{f}}$ et $\delta_{\mathbf{0}}$ dépendent principalement des caractéristiques de cet étage, dont l'étude est donc essentielle.

2. Choix de l'étage d'entrée. Caractéristiques des M.O.S.T. utilisés. - 2.1. GÉNÉralités. $-A$ priori, trois types de composants présentent les qualités requises de faible courant d'entrée et d'isolement important. Ge sont :

- les tubes électromètres,

- les condensateurs vibrants,

- les transistors à effet de champ à porte isolée (transistors à structure Métal-Oxyde-Semiconducteur ou M.O.S.T.).
Nous avons choisi ces derniers pour leur faible encombrement et l'homogénéité de leur structure qui les désignent tout particulièrement pour satisfaire les exigences d'ordre mécanique imposées à l'instrumentation spatiale. Ils présentent par ailleurs, par rapport aux deux premiers types de dispositifs signalés, l'avantage de ne nécessiter ni chauffage ni oscillateur. L'étude complète de ses caractéristiques électriques va cependant permettre de mieux définir les conditions d'emploi du transistor M.O.S. utilisé $\left({ }^{1}\right)$ dont la coupe schématique est représentée sur la figure 3 .

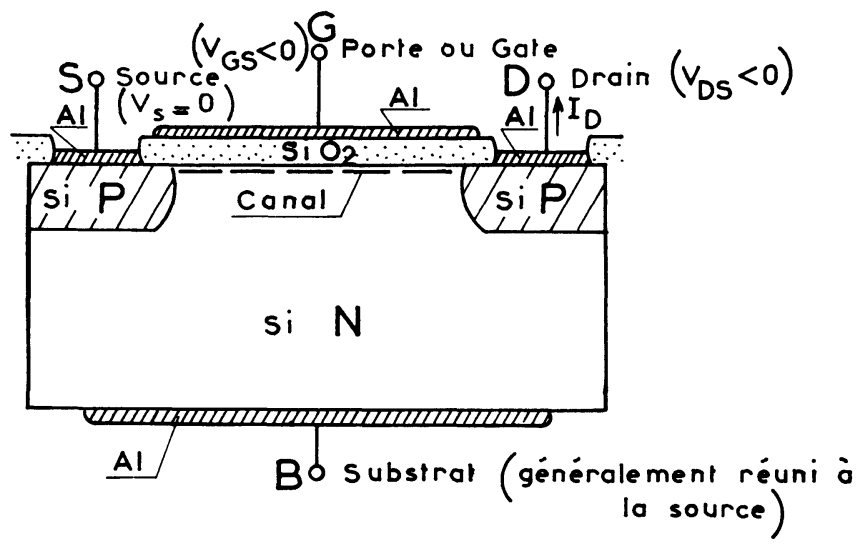

FIG. 3.

Coupe schématique d'un M.O.S.T. du type P-N-P

Ce composant solide fonctionne par effet de champ, la modulation de la conductibilité entre les électrodes source et drain s'effectuant par l'application d'un champ électrique aux bornes de la couche isolante de silice $\mathrm{SiO}_{2}[1,2,3]$.

2.2. Courant d'entrée. Isolement. - La mesure de ces paramètres [3] a montré :

- D'une part, l'existence d'un courant d'entrée extrêmement faible et diminuant avec le temps; ce courant, dû probablement aux phénomènes de polarisation lente des diélectriques, est inférieur à $10^{-16} \mathrm{~A}$ après une heure de fonctionnement.

- D'autre part, une assez grande dispersion, pour divers composants, des valeurs de la résistance d'isolement $R_{\mathrm{f}}$ entre l'électrode de commande et les autres électrodes $\left(10^{13}\right.$ à $\left.10^{16} \Omega\right)$; en fait, il est pratiquement certain que l'on mesure ainsi les fuites entre connexions à la traversée des boîtiers.

2.3. Glaquage du diélegtrique D'entrée. - Une surtension entre l'électrode-porte et l'une quelconque des autres électrodes provoque, lorsqu'elle entraîne un champ électrique suffisamment élevé, la dégradation de la couche de silice et l'apparition d'un courant d'entrée important rendant le composant inutilisable

(1) M.O.S.T. FI 100. 
pratiquement. Cette défaillance peut être provoquée par des charges électriques s'accumulant sur la connexion d'entrée.

2.4. Comportement sous irradiation. - De nombreuses études sont actuellement en cours pour déterminer le comportement des transistors M.O.S. soumis à l'effet des radiations $[4,5,6]$. Le groupe « CapteursComposants-Fiabilité » du Laboratoire de Génie Électrique a pour sa part étudié l'action des électrons de $1 \mathrm{MeV}$ et surtout celle des rayons $\mathrm{X}$. Les résultats détaillés et les hypothèses avancées figurent sur les références. Nous allons seulement mentionner les résultats obtenus lors d'irradiation par rayons $\mathrm{X}$, qui sont pratiquement les seuls rayonnements à prendre en considération lors des missions à altitude relativement basse.

Sur la figure 4, on constate la translation des carac-

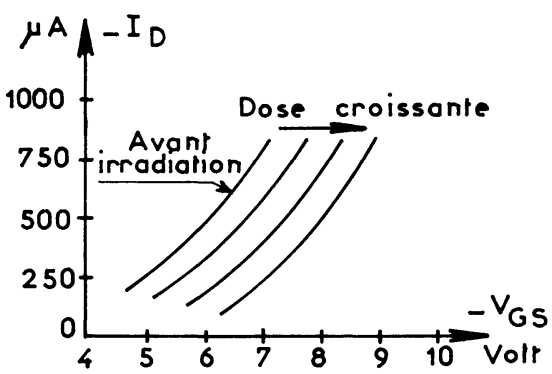

FIG. 4. - Évolution des caractéristiques de transfert d'un M.O.S.T. irradié par des rayons X de $215 \mathrm{keV}$.

téristiques de transfert; la pente du composant n'est donc pratiquement pas affectée par l'irradiation.

La figure 5 reproduit l'évolution de la tensionporte, à courant-drain et tension-drain constants, en

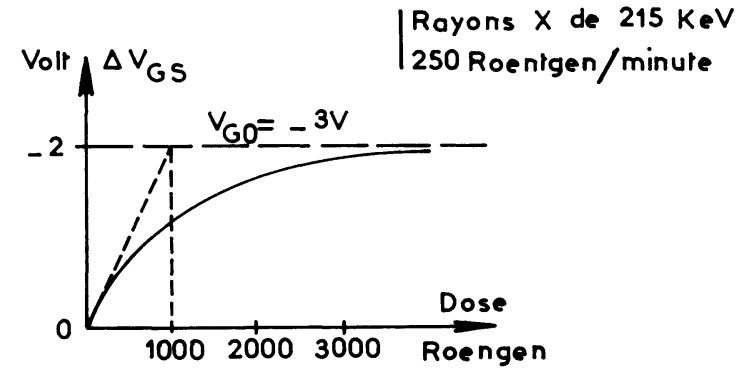

FIG. 5. - Évolution de la tension-porte (à courant et tension-drain constants) en fonction de la dose.

fonction de la dose absorbée. La sensibilité est maximale en début d'irradiation (les dérives mesurées sont comprises entre 1 et $5 \mathrm{mV} /$ Rœntgen), puis s'atténue.

Le palier de saturation n'est fonction que des polarisations du composant. Ce dernier résultat est important en pratique : on peut réduire considérablement la sensibilité d'un M.O.S.T. aux radiations en le préirradiant dans les conditions de polarisations qui correspondent exactement aux conditions prévues pour son utilisation.
2.5. ÉVOlution LENTE DES GARAGTÉRISTIQUES. Le transitoire de mise en route correspond à une tension $\Delta V_{\mathrm{GS}}$ ramenée sur la porte de l'ordre de $10 \mathrm{mV}$ pendant $15 \mathrm{mn}$ environ, puis la dérive s'atténue au cours du temps. Ce phénomène est réversible. Cependant, pour que le transitoire se reproduise, il n'est pas nécessaire de supprimer totalement la polarisation du composant. Il suffit que soit appliquée sur l'électrodeporte une impulsion de durée et d'amplitude telles qu'il y ait blocage du courant-drain par suppression du canal de conduction à l'interface diélectrique substrat [7]. Nous verrons au $\S 4.2 .1$ les moyens mis en œuvre pour nous affranchir de ce phénomène qui représente un grave inconvénient pratique, puisqu'un parasite d'origine électromagnétique peut le déclencher à instant quelconque.

3. Obtention d'une réponse logarithmique. 3.1. Relations et montages fondamentaux. - Pour constituer le circuit de contre-réaction, il nous a paru préférable également d'orienter notre étude vers la possibilité d'utiliser des composants « solides » et en particulier des éléments à semiconducteurs.

Des résultats expérimentaux antérieurs [8] confirment dans la gamme de courants $\left(10^{-9} \mathrm{~A}, 10^{-4} \mathrm{~A}\right)$ des résultats théoriques [9] conduisant aux expressions du courant collecteur et du courant émetteur de transistors (relations (4) et (5)). Nous avons poursuivi l'étude de ces paramètres pour les très faibles niveaux d'injection qui nous intéressent :

$$
\begin{aligned}
I_{\mathrm{c}}= & I_{\mathrm{c}_{1}}(T)\left[\mathrm{e}^{\frac{V_{\mathrm{eb}}}{U_{\mathrm{T}}}}-1\right] \\
& +I_{\mathrm{c}_{\mathrm{a}}}(T)\left[\mathrm{e}^{\frac{V_{\mathrm{eb}}}{U_{\mathrm{T}}}}-1\right]+I_{\mathrm{sc}}\left(V_{\mathrm{cb}}\right)+I_{\mathrm{c}_{0}}^{\prime}\left(V_{\mathrm{cb}}\right) \\
I_{\mathrm{e}}= & I_{\mathrm{e}_{1}}(T)\left[\mathrm{e}^{\frac{V_{\mathrm{eb}}}{U_{\mathrm{T}}}}-1\right] \\
& +I_{\mathrm{e}_{2}}(T)\left[\mathrm{e}^{\frac{V_{\mathrm{eb}}}{U_{\mathrm{T}}}}-1\right]+I_{\mathrm{se}}\left(V_{\mathrm{eb}}\right)+I_{\mathrm{RG}}\left(V_{\mathrm{eb}}\right)
\end{aligned}
$$

- $I_{\mathrm{c}}$ : courant collecteur,

- $I_{\mathrm{e}}$ : courant émetteur,

- $T$ : température absolue,

- $U_{\mathbf{T}}$ : tension thermodynamique à la température absolue $T$,

- $V_{\mathrm{eb}}:$ tension émetteur-base,

- $V_{\mathrm{cb}}:$ tension collecteur-base,

$-I_{\mathrm{c}_{1}}(T), I_{\mathrm{c}_{\mathrm{a}}}(T), I_{\mathrm{e}_{1}}(T), I_{\mathrm{e}_{\mathrm{a}}}(T)$ : fonctions de $T$, dont les valeurs à $T$ donnée ne dépendent que du composant considéré,

- $I_{\mathrm{sc}}\left(V_{\mathrm{cb}}\right)$ : courant de fuite en surface entre collecteur et base : $I_{\mathrm{sc}}(0)=0$,

- $I_{\mathrm{c}_{0}}^{\prime}\left(V_{\mathrm{cb}}\right)$ : courant de génération dans la charge d'espace collecteur-base ; $I_{\mathbf{c}_{0}}^{\prime}(0)=0$,

- $I_{\mathrm{se}}\left(V_{\mathrm{eb}}\right)$ : courant de fuite en surface entre émetteur et base,

- $I_{\mathrm{RG}}\left(V_{\mathrm{eb}}\right)$ : courant de génération-recombinaison au niveau de la base et de la connexion d'émetteur. 
Ces quatre derniers termes notés « courants parasites » ne suivent pas la loi en $\left(\mathrm{e}^{\frac{v}{U_{\text {T }}}}\right)$ [10].

Les mesures effectuées [3] ont porté sur la détermination des caractéristiques des transistors plans de faibles dimensions à surface passivée qui, dans l'état actuel de la technologie, présentent les moindres courants parasites. En effet, si ces derniers sont supposés nuls, les relations (4) et (5) conduisent pour $V_{\mathrm{cb}} \equiv 0$ à

$$
\begin{aligned}
I_{\mathrm{c}} & =I_{\mathrm{c}_{1}}\left[\mathrm{e}^{\frac{V_{\mathrm{eb}}}{U_{\mathrm{T}}}}-1\right] \\
I_{\mathrm{e}} & =I_{\mathrm{e}_{1}}\left[\mathrm{e}^{\frac{V_{\mathrm{eb}}}{U_{\mathrm{T}}}}-1\right] . \\
\text { Lorsque } \mathrm{e}^{\frac{V_{\mathrm{eb}}}{U_{\mathrm{T}}}} \gg 1: & 1: \\
V_{\mathrm{eb}} & =U_{\mathrm{T}}\left[\log I_{\mathrm{c}}-\log I_{\mathrm{c}_{1}}\right] \\
V_{\mathrm{eb}} & =U_{\mathrm{T}}\left[\log I_{\mathrm{e}}-\log I_{\mathrm{e}_{1}}\right] .
\end{aligned}
$$

Théoriquement, et dans les conditions particulières que nous avons indiquées, deux structures fondamentales d'amplificateurs sont donc valables ( fig. 6 et 7 ).

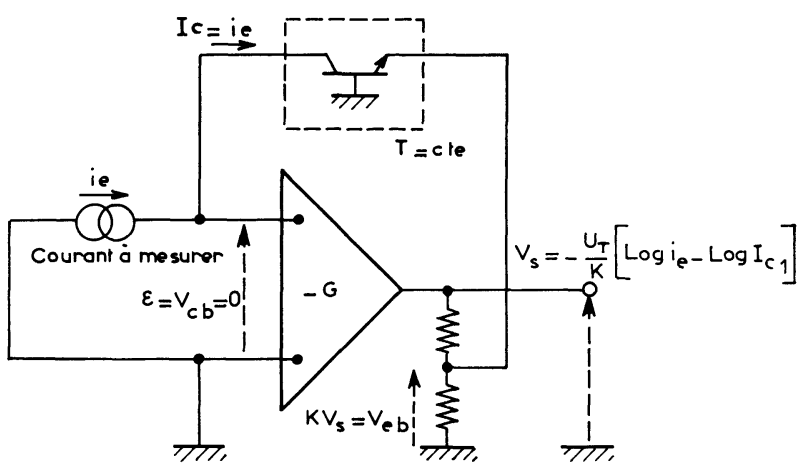

FIG. 6.

Utilisation de la caractéristique $V_{\mathrm{eb}}\left(I_{\mathrm{e}}\right)$ à $V_{\mathrm{cb}}=0$.

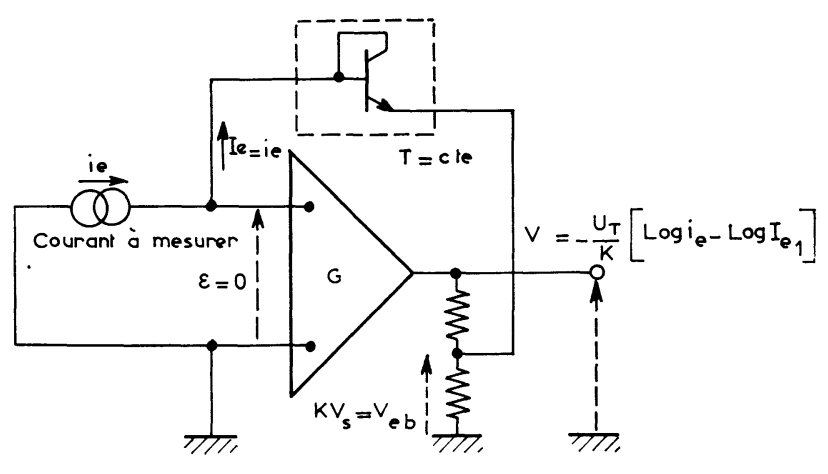

FIG. 7.

Utilisation de la caractéristique $V_{\mathrm{eb}}\left(I_{\mathrm{e}}\right)$ à $V_{\mathrm{eb}}=0$.

Elles utilisent comme élément de contre-réaction un transistor maintenu à température constante.

En pratique, seule la mesure fine des caractéristiques des transistors nous a permis de choisir le montage le mieux adapté, compte tenu des performances de la chaîne directe.

3.2. Étude du gourant collegteur. - Dans un premier temps, la mesure de la variation de la tension émetteur-base en fonction du courant collecteur, à tension collecteur-base nulle, a confirmé la relation exponentielle (8).

En coordonnées semi-logarithmiques, pour des ten-

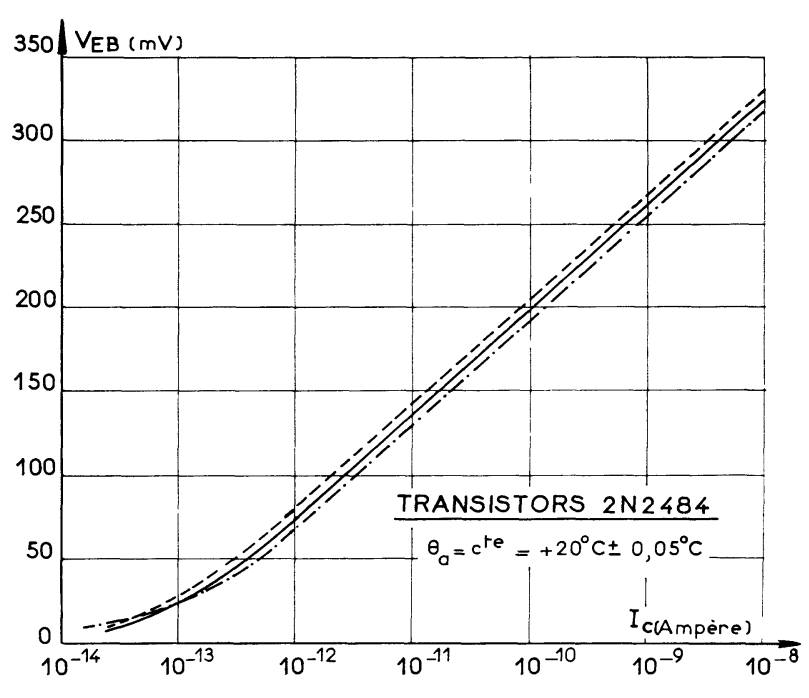

FIG. 8. $-V_{\mathrm{eb}}\left(I_{\mathrm{e}}\right)$.

sions $V_{\mathrm{eb}}>100 \mathrm{mV}$, soit environ $4 \times U_{\mathrm{T}=296 \mathrm{\circ K}}$, les caractéristiques obtenues sont rigoureusement linéaires, de pente égale pour tous les transistors étudiés. Cette pente est $\frac{U_{\mathrm{T}}}{\log \mathrm{e}}$ par décade (fig. 8).

Le second type de résultats expérimentaux concerne la sensibilité du courant collecteur, à tension émetteurbase nulle, en fonction de la tension collecteur-base et pour les faibles valeurs de cette dernière. On a trouvé pour de nombreux composants $\left({ }^{2}\right)$ :

$$
\begin{aligned}
& \left(\frac{\Delta V_{\mathrm{cb}}}{\Delta I_{\mathrm{c}}}\right)_{V_{\mathrm{eb}}=0, V_{\mathrm{cb} \ll}} \lesssim 4 \times 10^{10} \Omega \text { à }+20^{\circ} \mathrm{C} \\
& \left(\frac{\Delta V_{\mathrm{cb}}}{\Delta I_{\mathrm{c}}}\right)_{V_{\mathrm{eb}}=0, V_{\mathrm{eb} \ll}} \lesssim 10^{9} \Omega \text { à }+60^{\circ} \mathrm{C} .
\end{aligned}
$$

3.3. Étude DU Gourant ÉMETTEUR. - Une étude expérimentale préliminaire a permis de noter un écart important entre la caractéristique réelle et la caractéristique théorique déduite de la relation (9). Une détermination plus précise de cet écart est obtenue par la mesure simultanée de $I_{\mathrm{c}}, V_{\mathrm{eb}}$ et $I_{\mathrm{b}}=I_{\mathrm{e}}-I_{\mathrm{c}}$ à $V_{\mathrm{cb}}=0$.

La caractéristique $\log I_{\mathrm{c}}\left(\log I_{\mathrm{b}}\right)$ qui peut être appro-

(2) Types 2N 2484 et FM 2484. 


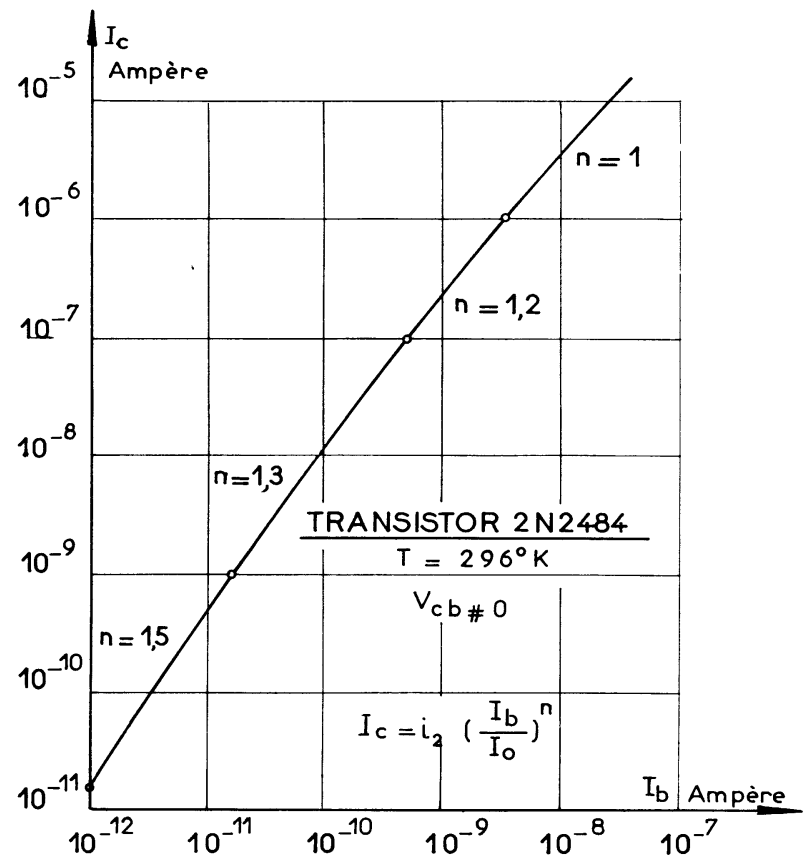

FIG. 9. - $\log I_{\mathrm{c}}\left(\log I_{\mathrm{b}}\right)$.

chée par une succession de segments de pente $n$ ( fig. 9) conduit à une expression du courant émetteur de la forme :

$$
I_{\mathrm{e}}=I_{\mathrm{c}}\left[1+I_{0} \frac{\frac{1-n}{n}}{A^{1 / n}}\right]
$$

où $n, A$ et $I_{0}$ sont des constantes dans chaque intervalle considéré.

La mesure précédente constitue donc un critère de choix des composants les mieux adaptés, quant à l'utilisation de la jonction émetteur-base, en vue de la réalisation d'un amplificateur logarithmique (cf. fig. 7).

Pour les très faibles courants, les courbes $\left[I_{\mathrm{e}}\left(V_{\mathrm{eb}}\right)\right]_{V_{\mathrm{cb}}=0}$ sont linéaires. On a obtenu :

$$
\begin{aligned}
& \left(\frac{\Delta V_{\mathrm{eb}}}{\Delta I_{\mathrm{e}}}\right)_{V_{\mathrm{eb}=0}} \lesssim 5 \times 10^{11} \Omega \text { à }+20^{\circ} \mathrm{C} \\
& \left(\frac{\Delta V_{\mathrm{eb}}}{\Delta I_{\mathrm{e}}}\right)_{V_{\mathrm{eb}=0}} \lesssim 10^{10} \Omega \text { à }+60^{\circ} \mathrm{C} .
\end{aligned}
$$

3.4. Choix DU GIRGUit DE GONTRE-RÉAGTION. - Les circuits représentés sur les figures 6 et 7 supposaient que la chaîne directe était sans bruit ni dérive. Il n'en est pas ainsi en réalité et les résultats (11) et (14) démontrent que le montage utilisant la caractéristique relative au courant émetteur provoque une erreur en courant environ dix fois plus faible que l'utilisation du transistor en tripôle dont la réponse est cependant plus proche de la loi logarithmique idéale.

Sacrifiant la reproductibilité de la fonction de trans- fert de l'amplificateur au bénéfice d'une meilleure stabilité de cette caractéristique, nous avons choisi le montage représenté sur la figure 7 .

4. Minimisation des dérives ( fig. 10 et 11). - Les circuits les plus caractéristiques de l'amplificateur sont représentés en détail sur la figure 10. Les étages de la chaîne directe autres que l'étage d'entrée sont globalement figurés par l'amplificateur de tension A. Les transistors utilisés sont tous du type plan à surface

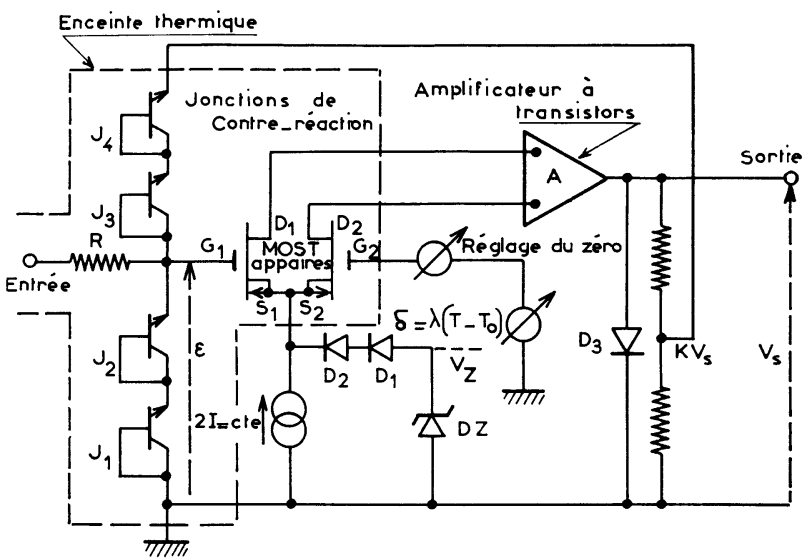

FIG. 10. - Structure de l'amplificateur.

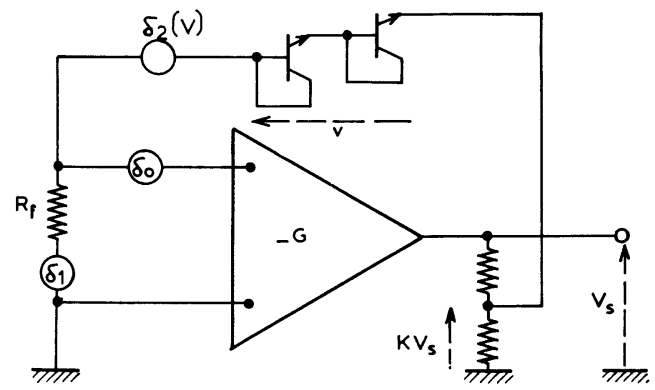

FIG. 11. - Bilan des dérives de l'amplificateur.

passivée. Les résistances sont à couches métalliques, en particulier celles qui influent le plus sur la stabilité du gain et les dérives ont un coefficient de température inférieur à $25 \times 10^{-6} \frac{\Omega}{\Omega^{\circ} \mathrm{C}}$.

L'énergie électrique est fournie aux circuits par l'intermédiaire d'alimentations stabilisées symétriques $\pm E\left(\frac{\Delta E}{E \Delta T}\right.$ inférieur à $\left.10^{-5} \mathrm{~V} / \mathrm{V}{ }^{\circ} \mathrm{C}\right)$.

4.1. Protection du M.O.S.T. D'entrée. - Cette protection est assurée d'une part par la mise en parallèle sur l'entrée des jonctions $J_{1}$ et $J_{2}$ (protection contre les surtensions négatives), d'autre part par l'existence des jonctions $\mathrm{J}_{3}$ et $\mathrm{J}_{4}$ de contre-réaction (protection contre les surtensions positives). En cas de surtension sur l'entrée, la résistance $R$, de forte valeur, placée 
entre l'entrée de l'amplificateur et la porte du premier M.O.S.T. limite le courant dans les jonctions, et la tension de cette porte ne peut dépasser une valeur prédéterminée, choisie suffisamment faible.

L'impédance ainsi mise en parallèle sur l'entrée doit être grande pour les faibles valeurs de la tension d'erreur existant en fonctionnement normal, et ainsi ne pas perturber les mesures par une trop importante diminution de $R_{\mathrm{f}}$. Actuellement, ce sont les jonctions planes au silicium, analogues à celles utilisées comme éléments logarithmiques, qui satisfont le mieux cet impératif.

Ayant ainsi pallié une défaillance éventuelle, nous nous sommes efforcé de réduire les contributions respectives de la chaîne directe et du circuit de contreréaction par les moyens que nous allons décrire.

4.2. Dérive En tension $\delta_{0}$ RAMENÉE A L'ENTRÉE DE LA GHAine DiRecte. - Tous les éléments de la chaîne directe n'interviennent pas au même titre dans la dérive globale $\delta_{0}$ puisque les contributions de chacun d'eux se ramènent à l'entrée, divisées par le gain des étages le précédant. Le premier étage est donc une des origines principales des dérives. Une structure symétrique constituée de deux M.O.S.T. appairés en caractéristiques et en dérives permet de réduire considérablement cet effet indésirable. Une nette amélioration des performances a été de plus obtenue par l'adjonction des circuits particuliers décrits dans les paragraphes suivants :

4.2.1. Transitoire d'évolution en fonction du temps. Il est très difficile pratiquement de choisir deux composants M.O.S. susceptibles de présenter dans le circuit réalisé la même évolution en fonction du temps. Le transitoire résiduel de « mise en route », dû principalement à ce déséquilibre, peut durer entre 3 et 5 heures. Après ce temps, la dérive ramenée à l'entrée est de l'ordre de $75 \mu \mathrm{V}$ par heure et s'atténue au cours du temps.

Pour utiliser au mieux cette dernière propriété de « saturation » de l'évolution en fonction du temps, un circuit spécial a été prévu, qui empêche le blocage du canal de conduction des M.O.S.T. $(\S 2.5)$ et donc la répétition fortuite du transitoire d'amplitude importante. Ce circuit (fig. 10), constitué de la diode Zéner $\mathrm{DZ}$ et des diodes $\mathrm{D}_{1}$ et $\mathrm{D}_{2}$, maintient, en association avec le circuit de protection décrit en 4.1 , la tension entre source et porte à une valeur choisie supérieure au seuil de blocage des M.O.S.T.

4.2.2. Influence des rayonnements électromagnétiques. La sensibilité de l'étage d'entrée est le terme prépondérant décrivant l'influence des rayonnements sur le dispositif amplificateur. La structure symétrique de cet étage conduit cependant à une contribution inférieure à $100 \mu \mathrm{V}$ par Rœntgen, pour des composants déséquilibrés de $10 \%$ sous certaines conditions de champ de polarisation porte-source.
4.2.3. Compensation des dérives thermiques. - La dérive thermique globale de la chaîne directe est annulée par le moyen d'un dipôle de compensation thermique dont la dérive $\delta$ ( fig. 10) est égale et opposée à la source de dérive équivalente ramenée à l'entrée de la chaîne directe. Le circuit utilise les propriétés de la dérive de la tension émetteur-base d'un transistor au Silicium en fonction de la température [11].

4.3. DÉrive $\delta_{2}$ DU GIRGuIT DE GONTRE-RÉAGTion. Des études $[8,12]$ conduisent à l'expression du coefficient de température d'une jonction de transistor au Silicium : le coefficient $\left(\frac{\Delta I_{\mathrm{e}}}{I_{\mathrm{e}} \Delta T}\right)_{V_{\mathrm{eb}}=\mathrm{C}^{\text {te }}}$ est fonction du niveau de polarisation, c'est-à-dire, dans notre exemple d'utilisation, du courant à mesurer. Ce résultat rend délicate la compensation de la dérive du circuit de contre-réaction par un moyen purement électronique.

La solution adoptée permet de s'affranchir plus simplement de la dérive $\delta_{2}$ en plaçant les éléments logarithmiques dans une enceinte maintenue à température constante. Le dispositif de régulation utilisée, issu d'une méthode mise au point au Laboratoire de Génie Électrique [13], est décrit en référence [3]. Si $\tau$ est le taux de régulation obtenu, $\tau=\frac{(\Delta T) \text { ambiante }}{(\Delta T) \text { interne }}$, compte tenu du fait que la dérive des éléments logarithmiques est maximale pour les très faibles courants et vaut alors environ $16 \%$ par degré, on peut écrire :

$$
\frac{\Delta i_{\mathrm{e}}}{i_{\mathrm{e}} \Delta T_{\mathrm{a}}}=\left(\delta_{2}\right)_{\mathrm{i}_{\mathrm{e}} \ll} \leqslant \frac{16}{100 \tau} .
$$

Quant à la sensibilité de ces éléments aux rayonnements, le problème ne se poserait que pour des doses élevées [10] que l'on ne peut actuellement appliquer aux M.O.S.T. sans dégradation.

4.4. Dérive $\delta_{1}$ Des jonctions DE PRotegtion. Cette dérive se traduit par une variation de la résistance de fuites $R_{\mathrm{f}}$. Le résultat (15) précédent est applicable. En effet, il est commode de placer tout le circuit haute impédance (jonctions de contre-réaction, jonctions de protection, M.O.S.T.) à l'intérieur de l'enceinte régulée en température. Cet ensemble constitue ainsi une « sonde » de très petites dimensions qui peut être placée à une certaine distance de l'amplificateur proprement dit et des circuits annexes : alimentations stabilisées, amplificateur de régulation thermique.

5. Performances d'un prototype expérimental. 5.1. Conditions EXPÉRIMENTALES. - Les études générales que nous avons effectuées ont permis la mise au point d'un prototype particulier d'amplificateur, dont nous avons déterminé les performances. Il convient de noter que les résultats mentionnés ci-après ont été obtenus dans des conditions très défavorables (tempé- 
rature de l'enceinte thermique fixée à $+60^{\circ} \mathrm{C}$ ) pour la sensibilité de l'amplificateur aux faibles courants $i_{\mathrm{e}}$ d'entrée (cf. relations (13) et (14)).

5.2. RÉgulation thermiQue. - Nous avons souligné en 4.3 le rôle fondamental joué par l'asservissement de température. L'enceinte thermique réalisée a une résistance thermique de fuites de $25^{\circ} \mathrm{C} /$ watt à une pression ambiante de $760 \mathrm{~mm}$ de mercure, sa capacité calorifique étant de 80 joules $/{ }^{\circ} \mathrm{C}$.

L'amplificateur d'asservissement permet d'obtenir avec cette enceinte un taux de régulation de 250 et une réponse transitoire satisfaisante ( fig. 12).

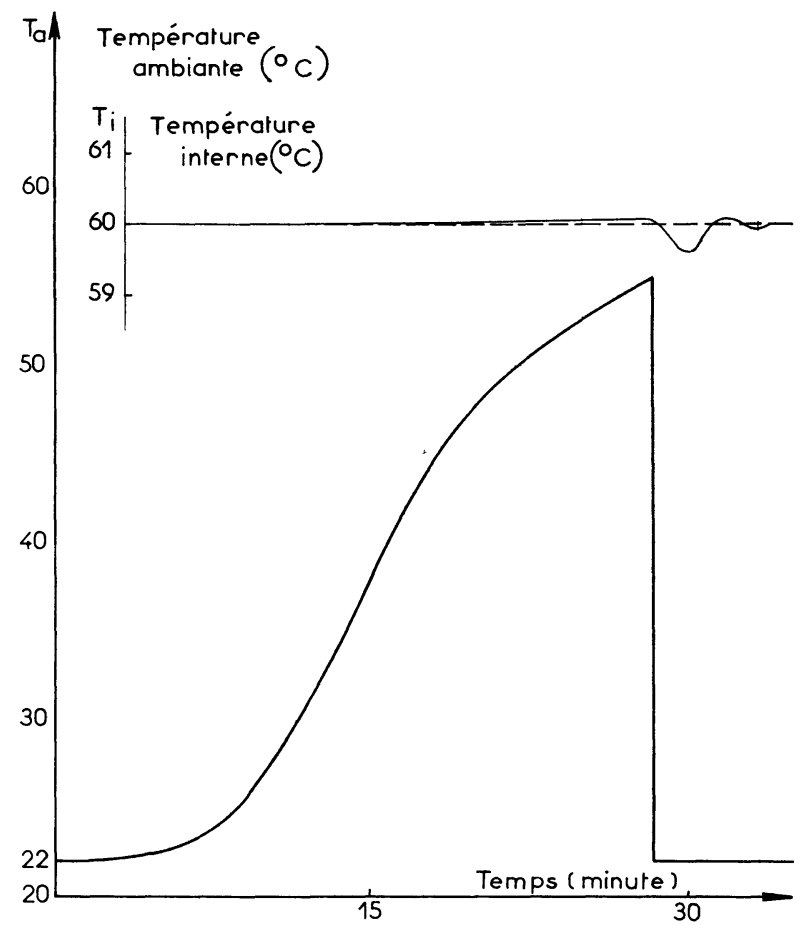

FIG. 12.

Caractéristiques de l'asservissement de température.
La consommation d'énergie, à la pression atmosphérique, est de $0,8 \mathrm{~W}$ pour $T_{\mathrm{a}}=+40^{\circ} \mathrm{C}$ et $1,6 \mathrm{~W}$ pour $T_{\mathrm{a}}=+20^{\circ} \mathrm{C}$.

5.3. Courbe D'Étalonnage DE L'Amplificateur (fig. 13). - Le rapport $K(\S 1)$ permet d'ajuster la

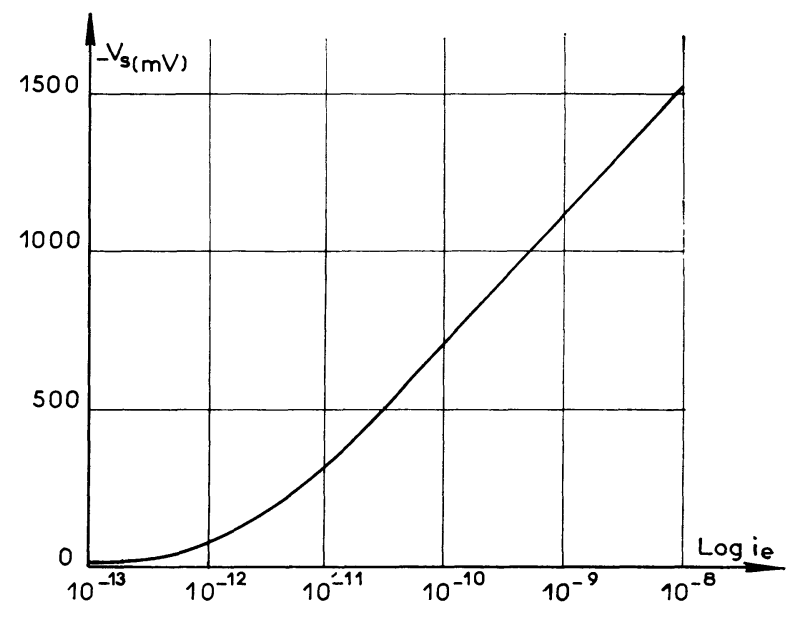

FIG. 13. - Courbe d'étalonnage.

pente de la caractéristique de manière à ce que la gamme des signaux d'entrée corresponde à une gamme de sortie normalisée. Dans notre exemple, nous nous sommes limité à $10^{-8} \mathrm{~A}$ en valeur supérieure, mais les jonctions des transistors FM 2484 utilisés présentent une caractéristique parfaitement logarithmique jusqu'à près de $1 \mathrm{~mA}$.

5.4. DÉRIVEs. BRUIT DE FOND. - Dérive temporelle inférieure à $10^{-15} \mathrm{~A} /$ heure après 24 heures de fonctionnement. - Action des rayonnements ionisants : 10-14 A/Rœntgen. - Action de la température : la figure 14 reproduit la réponse de l'amplificateur à un échelon thermique, pour une impédance de source infinie et dans le cas le plus défavorable (courant d'entrée nul). La dérive statique, pour

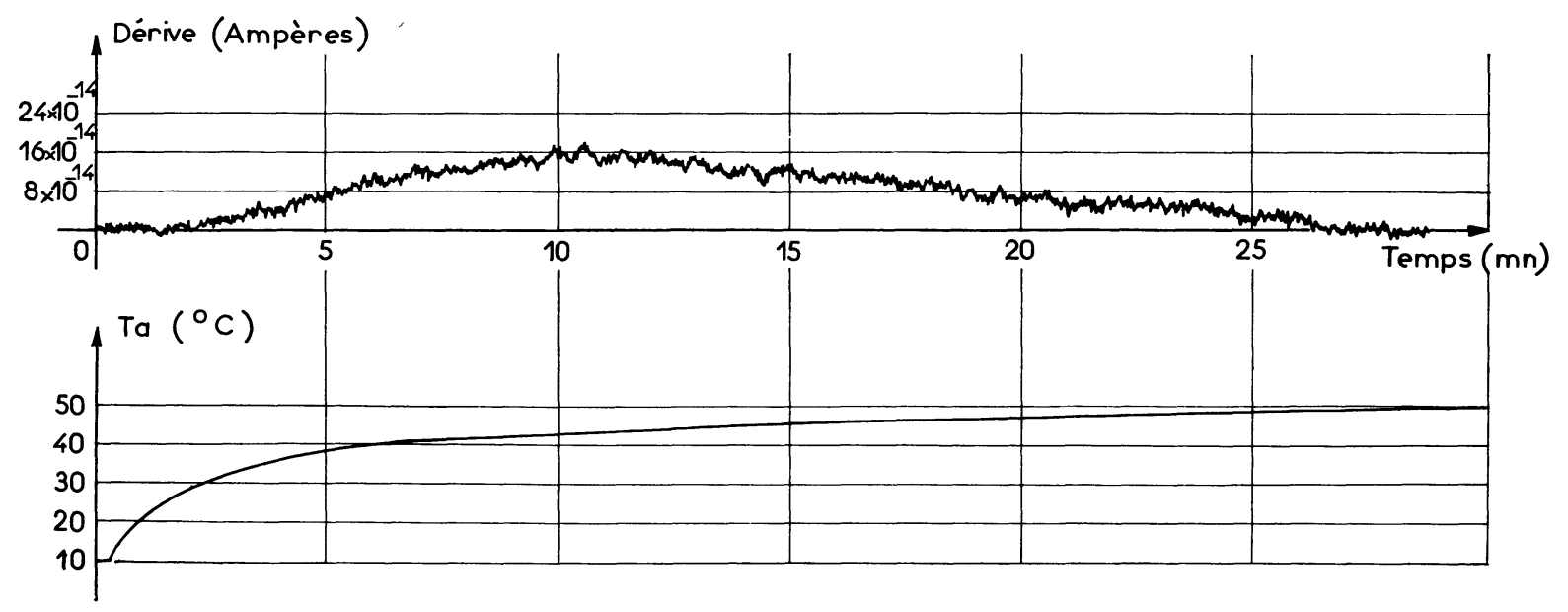

Frg. 14. - Réponse de l'amplificateur à un échelon de température. 
une variation de $40^{\circ} \mathrm{C}$, est inférieure au bruit de fond. - Bruit de fond dans la gamme $f<1 \mathrm{~Hz}$ : $3 \times 10^{-14} \mathrm{~A}$ crête à crête pour $i_{\mathrm{e}}=0 ; 1,5 \times 10^{-14} \mathrm{~A}$ crête à crête pour $i_{\mathrm{e}}=10^{-11} \mathrm{~A}$.

5.5. Constante de temps. - 20 à 60 millisecondes pour $i_{\mathrm{e}}=10^{-12}$ A. -30 microsecondes pour $i_{\mathrm{e}}=10^{-8} \mathrm{~A}$.

Conclusion. - Les résultats de nos études sur les caractéristiques de transistors M.O.S. montrent la possibilité de concevoir des amplificateurs électromètres utilisant ce type de composants nouveaux comme étage d'entrée. L'existence d'un phénomène de dérive lente a conduit à la mise en place d'un circuit de protection qui limite en général les applications à la mesure de courants supérieurs à $10^{-13} \mathrm{~A}$.

Compte tenu des propriétés électriques de l'étage d'entrée, nous avons démontré l'intérêt d'utiliser les jonctions émetteur-base de transistors plans au Silicium de petite géométrie pour assurer la réponse logarithmique sur une grande gamme de courants.
Les réalisations que l'on envisage peuvent être rendues relativement insensibles aux contraintes thermiques et mécaniques. De plus, la structure entièrement solide des amplificateurs ainsi définis augure une relative facilité d'intégration. Ces qualités sont appréciables en instrumentation spatiale.

La sensibilité des structures M.O.S. aux rayonnements constitue cependant à l'heure actuelle l'inconvénient majeur quant à l'utilisation de tels amplificateurs pour des missions spatiales de longue durée dans les zones dangereuses de radiations. Des recherches actuellement en cours permettent d'espérer une diminution de cette sensibilité d'un facteur dix et plus, mais d'ores et déjà le champ d'application des amplificateurs proposés peut recouvrir un grand nombre d'expériences embarquées, la dérive globale après stabilisation étant inférieure à $10^{-13} \mathrm{~A}$ pendant 18 mois à $400 \mathrm{~km}$ d'altitude.

Manuscrit reçu le 30 septembre 1966.

\section{BIBLIOGRAPHIE}

[1] Hofstein (S. R.), Heiman (F. P.), Proc. of the I.E.E.E., septembre 1963, p. 1190-1202.

[2] SAH (C. T.), I.E.E.E. Trans. on electron devices ED11, juillet 1964, p. 324-345.

[3] LIÉGEOIS (A.), Thèse de Docteur-Ingénieur, Faculté des Sciences de Toulouse, 16 juin 1966.

[4] ANDRÉ (B.), ESTTÈVE (D.), MARTinot (H.), SIMONNF (J.), Communication, Session S.F.E.R., Lyon, novembre 1965.

[5] Glotitin (P.), Communication, Journée d'étude S.F.E.R., 30 mars 1966.

[6] Giralt (G.), ANdRÉ (B.), Simonne (J.), EsTì̀ve (D.), Electronics letters, juin 1966, p. 209-210.

[7] Giralt (G.), André (B.), Simonne (J.), Estève (D.), C. R. Acad. Sc., Paris, juin 1964, 280, 6815-6818.
[8] Prajoux (R.), Thèse de Docteur-Ingénieur, Faculté des Sciences de Toulouse, 5 avril 1965.

[9] Gibbons (J. F.) et HoRn (H. S.), I.E.E.E. Trans. on circuit theory, septembre 1964, p. 378-384.

[10] BALdinger (E.) et LEZLINGER (M.), Solid-State Electronics, 1966, 9, 287-302.

[11] Giralit (G.), Communication, 4 es Journées internationales de calcul analogique, Brighton, septembre 1964.

[12] Lagasse (J.), Giralt (G.), Prajoux (R.), Automatisme, mars 1965, p. 93-101.

[13] Lagasse (J.), GrRali (G.), Brevet C.N.R.S., $\mathrm{n}^{\mathrm{o}}$ 1.352.441, mars 1964 . 